\title{
Helicobacter pylori-Induced Chronic Gastritis and Assessing Risks for Gastric Cancer
}

\author{
Gonzalo Carrasco $^{1}$ and Alejandro H. Corvalan ${ }^{2}$ \\ ${ }^{1}$ Department of Pathology, Mount Sinai School of Medicine, 1425 Madison Ave, New York, NY 10029, USA \\ ${ }^{2}$ Centre for Translational Research in Oncology (CITO) and Department of Hematology and Oncology, \\ Pontificia Universidad Catolica de Chile, Marcoleta 391, 8330074 Santiago, Chile
}

Correspondence should be addressed to Alejandro H. Corvalan; corvalan@med.puc.cl

Received 27 December 2012; Accepted 25 February 2013

Academic Editor: N. Ananthakrishnan

Copyright (C) 2013 G. Carrasco and A. H. Corvalan. This is an open access article distributed under the Creative Commons Attribution License, which permits unrestricted use, distribution, and reproduction in any medium, provided the original work is properly cited.

Chronic gastritis is an inflammation of the gastric mucosa and has multiple etiologies. Here we discuss the pathological alterations induced by Helicobacter pylori (HP) leading to chronic gastritis and the epigenetic bases underlying these changes. We review the histology of the normal gastric mucosa and overview the role of HP in the multistep cascade of GC. We attempt to define the role of the Operative Link for Gastritis Assessment (OLGA) staging system in assessing the risk of GC. The epigenetic bases of chronic gastritis, mainly DNA methylation, are presented through examples such as (i) the methylation of the promoter region of E-cadherin in HP-induced chronic gastritis and its reversion after HP eradication and (ii) the association of methylation of the promoter region of Reprimo, a p53-mediated cell cycle arrest gene, with aggressive HP strains in high risk areas for GC. In addition, we discuss the finding of RPRM as a circulating cell-free DNA, offering the opportunity for noninvasive risk assessment of GC. Finally, the integration of OLGA and tissue biomarkers, by systems pathology approach, suggests that severe atrophy has a greater risk for GC development if, in addition, overexpressed p73. This trial is registered with ClinicalTrials.gov NCT01774266.

\section{Introduction}

Since 1870, both human and veterinary pathologists have described bacterial infections based on the observation of tiny curved bacteria within gastric mucosa $[1,2]$. However, these organisms were dismissed as irrelevant contaminants. In 1947, when gastroscopy was first being used, Schindler deemed gastritis as "one of the most debated diseases of the human body" and predicted that its significance would be discussed "for some time to come" [3]. Schindler himself claimed that the "bacteriological etiology of chronic gastritis has not been convincingly proved in a single case" [3]. In 1984, Marshall and Warren proposed that chronic "idiopathic" gastritis had a bacterial cause, that is, Helicobacter pylori [4]. Their hypothesis was met with great skepticism. However, within a few years, the association between $H$. pylori gastritis, peptic ulcer, and gastric cancer came to be acknowledged and ultimately accepted [4]. For the purpose of this paper, we will focus mainly on the cascade of events produced by Helicobacter pylori infection leading to chronic changes in the gastric mucosa and the risk assessment for the development of gastric cancer. In addition, we will explore the epigenetic bases that underlie the changes of chronic gastritis.

\section{Normal Gastric Histology}

In order to recognize pathologic tissue responses in gastritis, it is essential to know the spectrum of normal gastric mucosa histology patterns. Normal gastric mucosa is formed by the epithelial/glandular and lamina propria components. The epithelial component consists of the foveolar epithelium, which is formed by tall columnar mucous cells with basally situated nuclei and supranuclear collections of closely packed small mucus globules that discharge their content onto the surface, forming an adherent protective lubricant layer that lines the lumen. The glandular component changes depending on its location in the stomach.

(1) Cardiac glands are limited to a narrow region of the stomach (the cardia) that surrounds the esophageal 


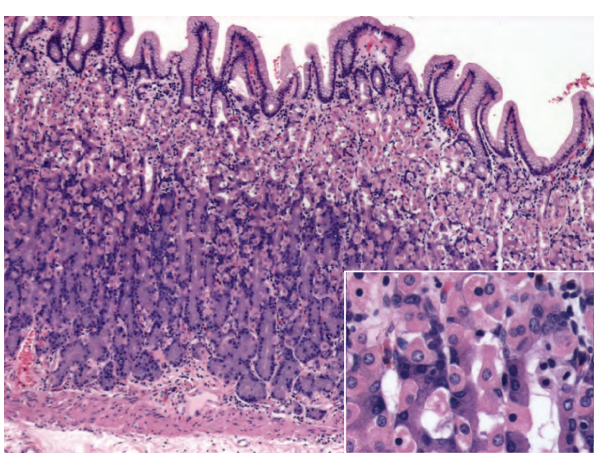

(a)

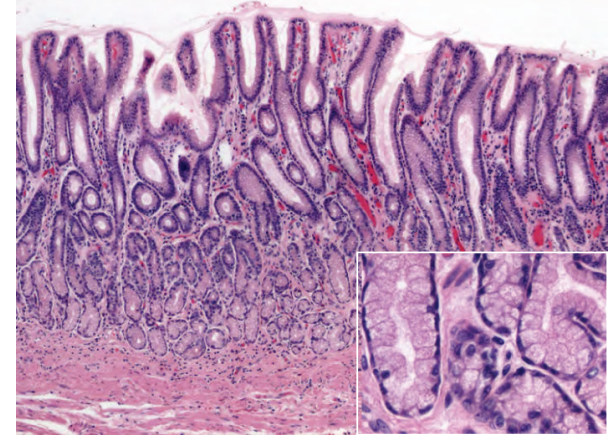

(b)

Figure 1: Normal gastric mucosa histology. ((a); H\&E 10x; square 40x) Fundic glands are simple, branched tubular glands that extend from the bottom of the gastric pits to the muscularis mucosae; the more distinctive cells are parietal cells. ((b); H\&E 10x; square 40x) Antral mucosa is formed by branched coiled tubular glands lined by secretory cells similar in appearance to the surface mucus cells.

orifice. They are tubular, somewhat tortuous, and occasionally branched, and are mainly formed by mucus-secreting cells, with occasional interspersed enteroendocrin cells.

(2) Fundic glands are present throughout the entire gastric mucosa, except for relative small regions occupied by cardiac and antral-pyloric glands. The fundic glands are simple, branched tubular glands that extend from the bottom of the gastric pits to the muscularis mucosae (Figure 1), and are formed by four functional types of cells: mucous neck cells, chief cells, enteroendocrine cells, parietal cells (also called oxyntic cells), and undifferentiated cells.

(3) Antral-pyloric glands are located in the pylori antrum (the part of the stomach between the fundus and the pylorus). They are branched coiled tubular glands and are lined by secretory cells similar in appearance to the surface mucus cells (Figure 1), suggesting a relative viscous secretion. Enteroendocrine cells are found interspersed within the gland epithelium along with occasional parietal cells.

The lamina propria is relatively scant and restricted to the limited spaces surrounding the gastric pits and glands. The stroma is composed of reticular fibers with associated fibroblasts and smooth muscle cells. It is also composed of lymphocytes, plasma cells, macrophages, and some eosinophils. The lymphocytes are predominantly immunoglobulin (Ig) A-producing B cells. IgG- and IgM-secreting cells are also present. Under normal conditions, intraepithelial lymphocytes are not present anywhere in the gastric mucosa. There is also a small number of lamina propria T cells, neutrophils, and mast cells. The lamina propria also contains capillaries, arterioles, and nonmyelinated nerve fibers. Small lymphoid aggregates, usually located in close proximity to the muscularis mucosae at the base of the lamina propria, especially at the corpus, could be present in normal gastric mucosa. In contrast, the presence of lymphoid aggregates with germinal centers is extremely rare in the mucosa of normal $H$. pylori negative adults [5].

\section{Histopathology of Helicobacter pylori-Induced Chronic Gastritis}

H. pylori is a microaerophilic gram-negative bacteria. The early phase of $H$. pylori infection elicits an acute inflammatory response that is either asymptomatic, or symptomatic with short-lived clinical manifestations such as nausea and vomiting, that evolve to a long-standing chronic gastritis [4]. Its prevalence goes from less than $15 \%$ in some populations to virtually $100 \%$, depending on socioeconomic status and country development [6]. In industrialized countries (Western Europe, United States, Canada, and Australia), exposure tends to occur later in life, which results in a lower percentage of infected adults. An average of $20 \%$ to $30 \%$ of adults is infected by age 50 [6] and the prevalence of $H$. pylori infection has been steadily declining in emerging countries, which is probably a reflection of improved sanitary conditions, as well as the widespread use of antibiotics. The foveolar epithelium produces a thick layer of mucus that plays a protective role. This mucus layer is the primary site for $H$. pylori colonization [7]; so, they characteristically attach to the surface mucous cells, but do not penetrate them. In chronic infection, $H$. pylori contacts the surface epithelial membrane, producing prominent epithelial degeneration $[8,9]$. The cells often become irregular and cuboidal in shape, showing a decrease in apical mucin content, as well as occasional "drop outs," which leaves small gaps in the epithelium and contributes to a ragged, disorderly appearance.

H. pylori preferentially colonize the antrum, but they may infect any part of the stomach where it causes gastritis. When treated, the bacteria migrate from the antrum to the corpus, decreasing the activity of antral gastritis. Marked neutrophilic infiltrates appear in the mucous neck region and lamina propria in early acute gastritis (Figure 2); when severe, they aggregate in the pit lumens to form pit abscesses. Both the neutrophils and the $H$. pylori destroy the epithelium, causing the mucous neck cells to proliferate in an effort to replace the dying cells. The regenerative pit bases are characterized by mucin loss, cytoplasmic basophilia, increased mitoses, and hyperchromatic nuclei that are sometimes severe enough to mimic dysplasia. 


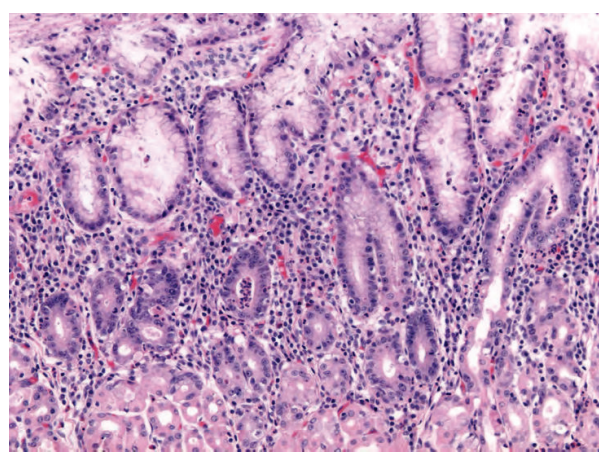

(a)

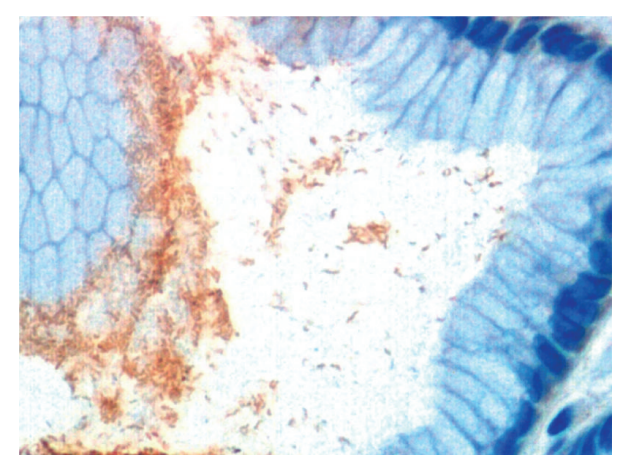

(b)

FiguRE 2: Early acute superficial gastritis. ((a); H\&E 20x) Marked neutrophilic infiltrates appear in the mucous neck region and lamina with a pit micoabscess. ((b); H. pylori immunostaining: rabbit polyclonal; clone $\mathrm{CH}-20$ 429, Novocastra; $40 \mathrm{x})$ Numerous $H$. pylori bacteria are present in the superficial foveolar epithelium.

Neutrophilic inflammation and the presence of lymphoid follicles with germinal centers are the two most distinctive histological features of $H$. pylori infection, and its eradication causes rapid neutrophil disappearance; thus their continued presence is considered a valuable indicator of therapeutic failure. The surface changes reverse rapidly, and the epithelial cells acquire their normal shape and spatial organization within a few days of $H$. pylori eradication. However, any atrophy that had developed remains, as did the lymphoid aggregates [10]. These features become permanent components of the once-infected gastric mucosa.

\section{Grading the Helicobacter pylori-Induced Chronic Gastritis: The Updated Sydney System}

The most widely used grading system for gastritis is the Updated Sydney System [11]. The system provides guidelines for generating systematic and uniform diagnostic reports. The goal of the Sydney System is to make gastric biopsy pathology reporting consistent, so that clinical studies can be performed and evaluated in a meaningful manner. The system classifies chronic gastritis on the basis of topography, morphology, and, when possible, etiology, into three broad categories: acute, chronic, and special (or distinctive). The biopsy protocol recommends that specimens from three compartments (i.e., antrum, incisura angularis, and corpus) should be separately designated when submitted to the pathology laboratory. Each relevant pathologic feature (density of $H$. pylori, intensity of neutrophilic and mononuclear inflammation, atrophy of the antrum and corpus, and intestinal metaplasia) should be graded on a standardized visual analogue scale (Figure 3). Each feature is assigned either a numeric or descriptive value: 0 for absent, 1 for mild, 2 for moderate, and 3 for marked (or severe). The values of each specimen are then averaged separately for each anatomic compartment (antrum and corpus). The next step is to document the degree of inflammation in the two main gastric compartments (antrum and corpus) and to determine whether the inflammation is similar in intensity (i.e., pangastritis) or more severe in either the antrum (antrumpredominant gastritis) or the corpus (corpus-predominant gastritis).

\section{Helicobacter pylori-Induced Chronic Gastritis and Multistep Cascade of Gastric Carcinogenesis}

In gastric cancer development, $H$. pylori-induced chronic gastritis is the first step of the so-called multistep cascade of gastric cancer. The cascade sequence of gastric carcinogenesis includes the nonatrophic chronic gastritis, multifocal atrophic gastritis, intestinal metaplasia, low-grade dysplasia (low-grade noninvasive neoplasia), high-grade dysplasia (high-grade noninvasive neoplasia), and invasive adenocarcinoma as described by Correa as the "human model of gastric carcinogenesis" [12]. This multistep model hypothesizes that the sequence of lesions reflects a dynamic process from a naïve inflammation caused by $H$. pylori infection to a fully malignant neoplasm of the stomach [13-16]. Independent epidemiological studies have confirmed that these entities are all linked through a sequential cause-effect relationship, thus supporting the concept of a human model for gastric carcinogenesis [17-19]. In a recent review and update of this model, it is also postulated that $H$. pylori is present not only in the first step of gastric mucosa inflammation but as an etiological factor in every step of the precancerous cascade [20]. In the first step, H. pylori infection targets normal mucosa with well-preserved gastric glands, by definition such gastritis is nonatrophic. At this point, it can be cured by clearing $H$. pylori infection or it may be evolving in two ways: it can remain as nonatrophic or it progresses in severity, leading to damage to the gastric glands, which may eventually disappear [20]. The progression depends on the interplay of three sets of etiological factors: infectious agent, host's genetic susceptibility and external environment; these determine the susceptibility and severity of outcome in the subset of individuals that develop clinical disease $[20,21]$. The presence of virulent factors in the infecting $H$. pylori strain is a known determinant factor of the outcome of the infection. Infection 


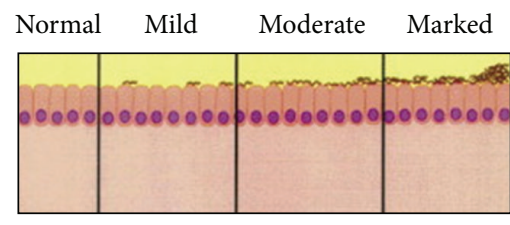

H. pylori

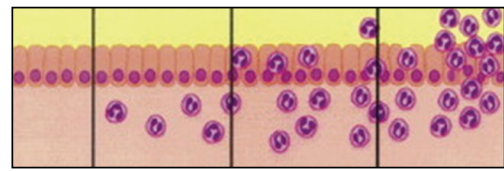

Neutrophils

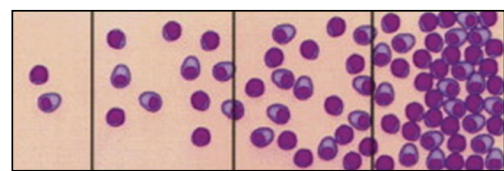

Mononuclear cells

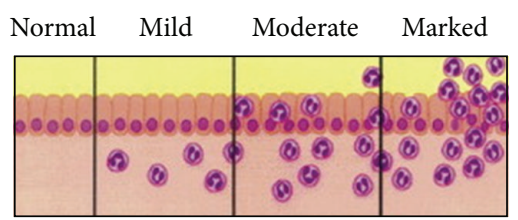

Atrophy: antrum

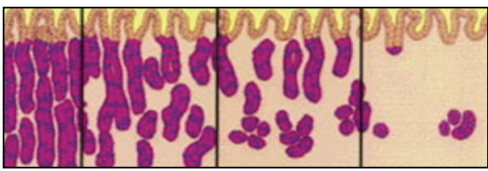

Atrophy: corpus

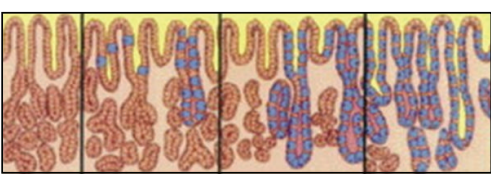

Intestinal metaplasia

Figure 3: The Updated Sydney System visual standardized visual analogue scale. Each feature is assigned either a numeric or descriptive value: 0 for absent, 1 for mild, 2 for moderate, and 3 for marked (or severe). Taken from Dixon et al. [11].

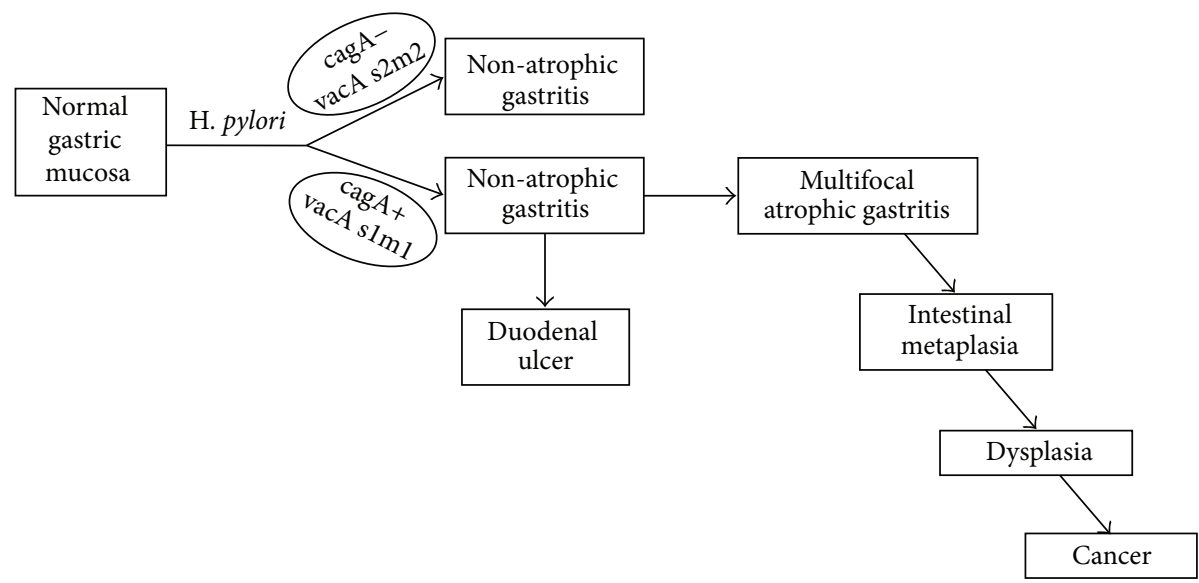

FIgURE 4: Multistep cascade of gastric cancer. This sequence begins with the infection of $H$. pylori to sequential steps of the precancerous cascades. Taken from Correa and Piazuelo [20].

with cag-positive vacA slm1 strains is associated with the development of gastric cancer, while cag-negative vacA s2m2 infection does not increase the risk of cancer and is associated to the persistency of nonatrophic gastritis (Figure 4) [20,22].

\section{Assessing Risks for \\ Gastric Cancer: The Operative Link for Gastritis Assessment (OLGA)}

The risk of malignant transformation of the lesions associated with the multistep cascade of gastric cancer is poorly defined. Long-term follow-up studies have shown a risk from $10 \%$ to $17 \%$ in the case of dysplasia [23-27]. For intestinal metaplasia, the risk assessment has conflicting results and therefore a limited clinical value [28-32]. The recently developed Operative Link for Gastritis Assessment (OLGA) staging system [33], through the evaluation of the extension and site of the atrophic changes, is an attempt to evaluate the risk of chronic gastritis to progress to intestinal metaplasia and gastric cancer [34-36]. Long standing H. pylori infection may lead to the loss of functional glands (atrophy) and replacement of the normal gland and foveolar epithelium by intestinal type cells (intestinal metaplasia) (Figure 5), the two main histological abnormalities invariable present as the background of gastric cancer [37]. The extent and site of the atrophic changes significantly correlate with cancer risk [38]. Two main types of atrophy can be recognized: one characterized by the loss of glands, accompanied by fibrosis or fibromuscular proliferation in the lamina propria, and the other characterized by the replacement of the normal (native) glands with metaplastic glands (i.e., glands that do not normally belong to that area) [39]. The degree of atrophy and metaplasia can be assessed with OLGA staging system for atrophy risk assessment [33]. This new system requires that specimens are taken according to the biopsy protocol 


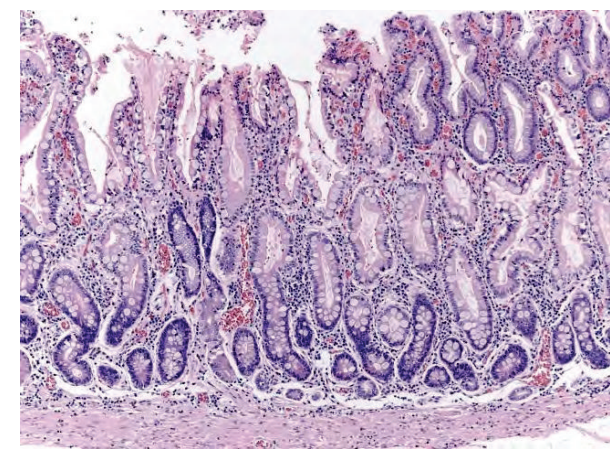

(a)

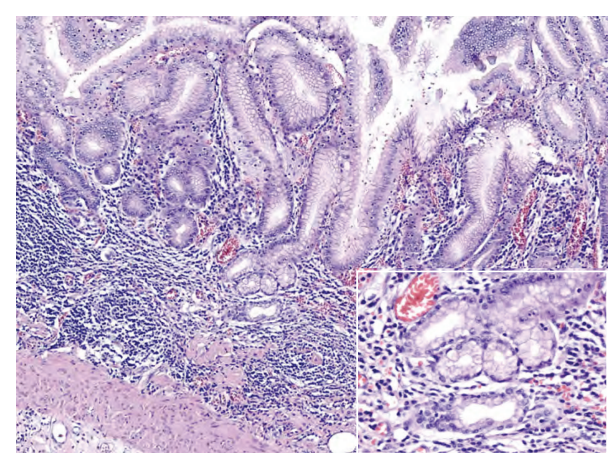

(b)

FIGURE 5: Atrophy is the loss of appropriate glands. ((a); H\&E 10x) Antral gastric mucosa with accentuated atrophy because replacement by extensive intestinal metaplasia. ((b); H\&E 10x; square 20x) Fundic-corporal gastric mucosa with extensive loss of gastric glands, partially replaced by pseudo-pyloric metaplasia.

TABLE 1: The OLGA staging frame. Atrophy is scored as the percentage of atrophic glands and scored on a four-tiered scale. No atrophy $(0 \%)=$ score 0 ; mild atrophy $(1-30 \%)=$ score 1 ; moderate atrophy $(31-60 \%)=$ score 2 ; 9 severe atrophy $(>60 \%)=$ score 3 . These scores $(0-3)$ are used in the OLGA staging assessment in each 10 compartment. Taken from Rugge et al., Dig Liver Dis 2011; 43:S373-84 with permission of Elsevier.

\begin{tabular}{|c|c|c|c|c|c|}
\hline & \multirow[b]{2}{*}{ Atrophy score } & \multicolumn{4}{|c|}{ Corpus } \\
\hline & & $\begin{array}{l}\text { No atrophy } \\
\text { (score } 0)\end{array}$ & $\begin{array}{l}\text { Mild atrophy } \\
\text { (score 1) }\end{array}$ & $\begin{array}{l}\text { Moderate atrophy } \\
\text { (score } 2)\end{array}$ & $\begin{array}{c}\text { Severe atrophy } \\
\text { (score } 3)\end{array}$ \\
\hline \multirow{4}{*}{ Antrum } & $\begin{array}{l}\text { No atrophy (score } 0 \text { ) } \\
\text { (including incisura angularis) }\end{array}$ & Stage 0 & Stage I & Stage II & Stage II \\
\hline & $\begin{array}{l}\text { Mild atrophy (score 1) } \\
\text { (including incisura angularis) }\end{array}$ & Stage I & Stage I & Stage II & Stage III \\
\hline & $\begin{array}{l}\text { Moderate atrophy (score 2) } \\
\text { (including incisura angularis) }\end{array}$ & Stage II & Stage II & Stage III & Stage IV \\
\hline & $\begin{array}{l}\text { Severe atrophy (score } 3 \text { ) } \\
\text { (including incisura angularis) }\end{array}$ & Stage III & Stage III & Stage IV & Stage IV \\
\hline
\end{tabular}

of the Sydney System, and that atrophy is scored in a fourtiered scale (0-3) according to the visual analogue scale of the Houston-updated Sydney system. The stage resulting from the combination of atrophic changes was assessed in the two mucosal compartments considered herein (Table 1).

\section{Assessing Risks for Gastric Cancer: The Epigenetic Bases of Chronic Gastritis}

The molecular bases of the multistep process of gastric carcinogenesis are highly relevant since it contributes greatly to assess risks of gastric cancer. Therefore, chronic gastritis should be understood as a disturbance in the balance between tumor suppressor genes and oncogenes. Many tumor suppressor genes have been identified in gastric cancer as well as chronic gastritis [40]. For example, inactivation of p53 tumor suppressor gene, E-cadherin, and DNA mismatch repair genes (hMSH2 and hMLH1) responsible for loss of heterozygosity $(\mathrm{LOH})$ and microsatellite instability (MSI) are well-recognized examples. However, multiple studies have shown that mutation and/or deletion is an infrequent mechanism of inactivating these well-established tumor suppressor genes [41]. In this scenario, epigenetic alterations, such as
DNA methylation, have been proposed as an alternative mechanism for inactivation of tumor suppressor genes [41]. DNA methylation is a process in which cytosines acquire a methyl group in $5^{\prime}$ position only if they are followed by a guanine (CpG site) [42]. Since DNA methylation has been considered as an excellent candidate to explain how environmental factors may increase the risk of cancer, it has been proposed as a key element for the early events of gastric carcinogenesis [43]. For example, Chan et al. [44] found that DNA methylation of the promoter region of Ecadherin has been associated with $H$. pylori infection. This association was independent of the age and/or type of gastritis [44]. Furthermore, the same authors [45] also demonstrate that $H$. pylori eradication with antibiotics reverses the DNA methylation of E-cadherin (Figure 6). Similarly, Maekita et al. [46] analyzed the effect of $H$. pylori infection on DNA methylation for multiple genes (HAND1, HRASLS, LOX, p16, P41ARC, and THBD) in $H$. pylori negative and positive healthy donors and gastric cancer patients. Among healthy donors, methylation levels were higher in $H$. pylori positives than in $H$. pylori negatives [46]. Taken together these data suggest that $H$. pylori infection induces DNA methylation mostly in premalignant conditions rather than gastric cancer itself [46]. Among other tumor suppressor genes inactivated 
Week 0

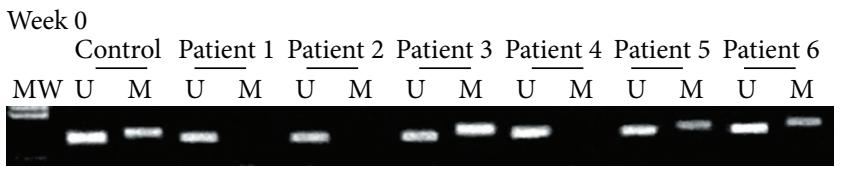

(a)
T ATGCGAGG TC GGG TGGGTGGGTCGTTAGTTT CG TTT T

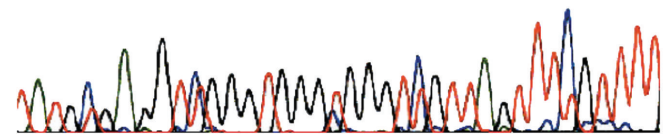

(b)
Week 6

Control Patient 1 Patient 2 Patient 3 Patient 4 Patient 5 Patient 6

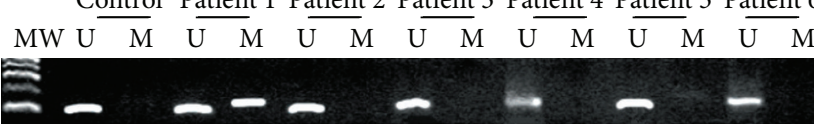

(c)

Week 6

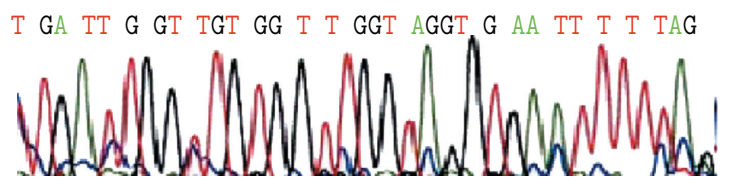

(d)

Figure 6: CpG island methylation pattern at the E-cadherin gene in gastric mucosa from patients with dyspepsia. (a) Before eradication of $H$. pylori (week 0), methylation was present in patients 3, 5, and 6. (b) The methylated product was confirmed by sequencing using the same methylated primer. (c) After eradication of $H$. pylori (week 6), methylation was not present in any patient. (d) The methylated product was again confirmed by sequencing using the same methylated primer. No methylated cytosine was seen. MW: molecular weight marker, U: unmethylated band, M: methylated band, red color: unmethylated cytosines converted to thymidine, blue color: methylated cytosines. Taken from Chan and Rashid [45].

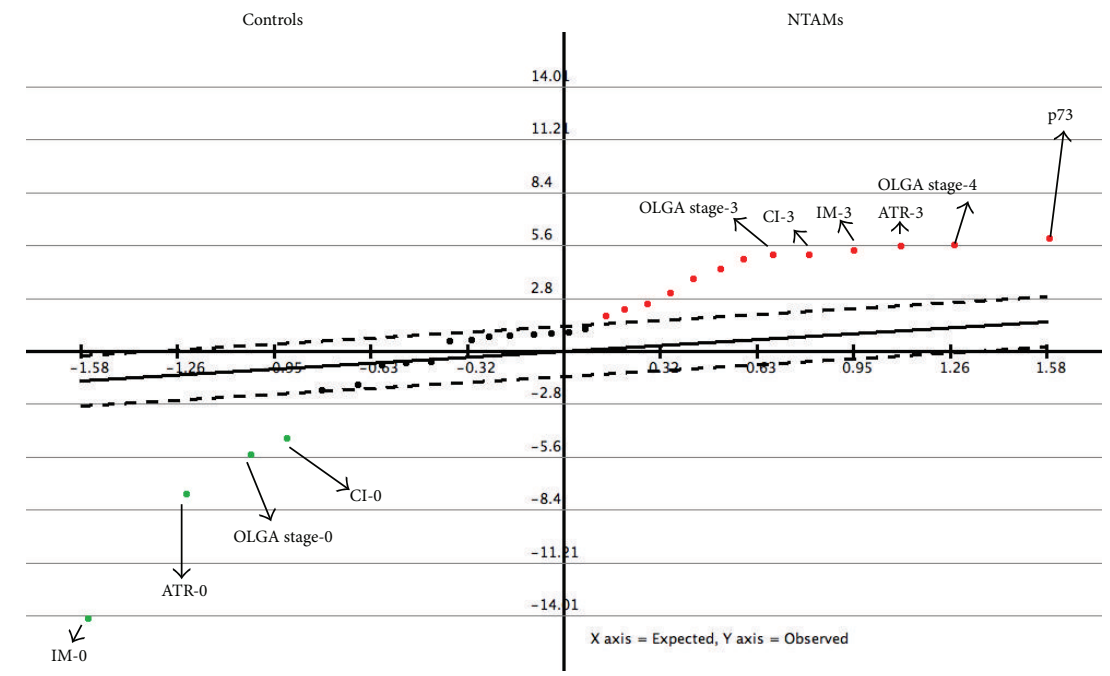

FIGURE 7: Serial analysis for microarray from nontumor adjacent mucosa (NTAM) and chronic gastritis controls. NTAM group is significantly characterized by the overexpression of p73, OLGA stages III to IV, and severe atrophy (ATR-3), intestinal metaplasia (IM-3), and chronic inflammation (CI-3) according to the Sydney System. Control group cases were significantly characterized by lack of intestinal metaplasia (IM-0), atrophy (ATR-0), and chronic inflammation (CI-0). False discovery rate $=0$. Taken from Carrasco et al., [51].

by DNA methylation, Reprimo (RPRM), a downstream mediator of p53-induced G2 cell cycle arrest [47], has been recently associated with more aggressive $H$. pylori strains (cag-positive, vacA s1 ml, and EPIYA polymorphisms) in Colombian residents from areas with high incidence of gastric cancer [48]. Furthermore, we have identified that DNA methylation of RPRM is not only found in gastric mucosa but also in the plasma of gastric cancer patients [49]. Therefore, this circulating cell-free DNA offers the opportunity for noninvasive assessing risks for gastric cancer. Since a recent meta-analysis suggests that among several candidates, circulating cell-free DNA of RPRM methylation is the most promising [50], this issue is currently under evaluation (Detection of Methylated Reprimo in Plasma for Asymptomatic Gastric Cancer, DEMRAC study. Finally, we have recently attempted to identify specific tissue biomarkers for assessing the risk of premalignant gastritis [51]. To this purpose, we evaluate the tissue overexpression, as a consequence of DNA hypomethylation, of several oncoproteins associated with gastric cancer including STAT1, p73, FHIT, p16INK4a, BRCA1, HSP90, and EGFR. These tissue biomarkers were compared with $H$. pylori and the OLGA staging system. As expected, severe atrophy and OLGA stage IV were the most relevant histological features of premalignant gastritis. Among tissue biomarkers, overexpression of p73 was the most relevant finding. Both data were integrated by systems pathology approach by performing Significance Analysis of Microarrays [52, 53]. This approach shows that p73 is stronger as a single variable when compared with OLGA stage IV (Figure 7). Therefore, we believe that gastritis 
with severe atrophy has a greater risk for developing gastric cancer if, in addition, overexpress p73 [51].

In summary the role of $H$. pylori in the development of chronic gastritis is not only associated to the bacteria itself but also to host and environmental factors. Assessing risks for gastric cancer can be achieved by the evaluation of clinical, morphological, and molecular factors. Among morphological criteria, atrophy and intestinal metaplasia can be evaluated via Sydney and OLGA approaches. Molecular factors that should be considered are mostly E-cadherin methylation, circulating cell-free DNA of RPRM methylation, and overexpression of p73. Finally, a system pathology approach allows integrating all these factors that might be useful in switching from an interpretive and subjective morphologically oriented approach to a more objective, evidence-based tissue marker approach.

\section{Acknowledgment}

This paper is supported by Grants nos. Fondef\#D09I1137 and Fondecyt\#1111014 from the Government of Chile to A. H. Corvalan.

\section{References}

[1] N. Figura and G. Oderda, "Reflections on the first description of the presence of helicobacter species in the stomach of mammals," Helicobacter, vol. 1, no. 1, pp. 4-5, 1996.

[2] M. Kidd and I. M. Modlin, "A century of Helicobacter pylori. Paradigms lost-paradigms regained," Digestion, vol. 59, no. 1, pp. 1-15, 1998.

[3] R. Schindler, Gastritis, William Heinmann, London, UK, 1947.

[4] B. J. Marshall and J. R. Warren, "Unidentified curved bacilli in the stomach of patients with gastritis and peptic ulceration," The Lancet, vol. 1, no. 8390, pp. 1311-1315, 1984.

[5] B. C. Jacobson, J. M. Crawford, and F. A. Farraye, "GI tract endoscopic and tissue processing techniques and normal histology in surgical pathology of the GI tract," in Liver, Biliary Tract and Pancreas, R. D. Odze and J. R. Goldblum, Eds., Elsevier, 2009.

[6] H. M. Malaty, "Epidemiology of Helicobacter pylori infection," Best Practice and Research, vol. 21, no. 2, pp. 205-214, 2007.

[7] T. Shimizu, T. Akamatsu, H. Ota, and T. Katsuyama, "Immunohistochemical detection of Helicobacter pylori in the surface mucous gel layer and its clinicopathological significance," Helicobacter, vol. 1, no. 4, pp. 197-206, 1996.

[8] F. Carneiro, E. Fonseca, and M. Sobrinho-Simoes, "Epithelial degeneration induced by Helicobacter pylori," Human Pathology, vol. 23, no. 11, pp. 1314-1315, 1992.

[9] K. M. Leung, P. K. Hui, W. Y. Chan, and T. M. M. Thomas, "Helicobacter pylori-related gastritis and gastric ulcer: a continuum of progressive epithelial degeneration," American Journal of Clinical Pathology, vol. 98, no. 6, pp. 569-574, 1992.

[10] R. M. Genta, G. M. Lew, and D. Y. Graham, "Changes in the gastric mucosa following eradication of Helicobacter pylori," Modern Pathology, vol. 6, no. 3, pp. 281-289, 1993.

[11] M. F. Dixon, R. M. Genta, J. H. Yardley, and P. Correa, "Classification and grading of gastritis. The updated Sydney system. International Workshop on the Histopathology of Gastritis, Houston 1994," The American Journal of Surgical Pathology, vol. 20, no. 10, pp. 1161-1181, 1996.
[12] P. Correa, "Chronic gastritis: a clinico-pathological classification," American Journal of Gastroenterology, vol. 83, no. 5, pp. 504-509, 1988.

[13] P. Correa, C. Cuello, E. Duque et al., "Gastric cancer in Colombia. III. Natural history of precursor lesions," Journal of the National Cancer Institute, vol. 57, no. 5, pp. 1027-1035, 1976.

[14] C. Cuello, P. Correa, W. Haenszel et al., "Gastric cancer in Colombia. I. Cancer risk and suspect environmental agents," Journal of the National Cancer Institute, vol. 57, no. 5, pp. 10151020, 1976.

[15] W. Haenszel, P. Correa, C. Cuello et al., "Gastric cancer in Colombia. II. Case control epidemiologic study of precursor lesions," Journal of the National Cancer Institute, vol. 57, no. 5, pp. 1021-1026, 1976.

[16] P. Correa, W. Haenszel, C. Cuello et al., "Gastric precancerous process in a high risk population: cohort follow-up," Cancer Research, vol. 50, no. 15, pp. 4737-4740, 1990.

[17] H. Ohata, S. Kitauchi, N. Yoshimura et al., "Progression of chronic atrophic gastritis associated with Helicobacter pylori infection increases risk of gastric cancer," International Journal of Cancer, vol. 109, no. 1, pp. 138-143, 2004.

[18] V. Conteduca, D. Sansonno, G. Lauletta, S. Russi, G. Ingravallo, and F. Dammacco, "H. pylori infection and gastric cancer: state of the art (Review)," International Journal of Oncology, vol. 42, no. 1, pp. 5-18, 2013.

[19] C. A. Gonzalez and A. Agudo, "Carcinogenesis, prevention and early detection of gastric cancer: where we are and where we should go," International Journal of Cancer, vol. 130, no. 4, pp. 745-753, 2012.

[20] P. Correa and M. B. Piazuelo, "The gastric precancerous cascade," Journal of Digestive Diseases, vol. 13, no. 1, pp. 2-9, 2012.

[21] R. M. Delahay and M. Rugge, "Pathogenesis of Helicobacter pylori infection," Helicobacter, vol. 17, supplement 1, pp. 9-15, 2012.

[22] C. A. González, C. Figueiredo, C. B. Lic et al., "Helicobacter pylori cagA and vacA genotypes as predictors of progression of gastric preneoplastic lesions: a long-term follow-up in a highrisk area in Spain," American Journal of Gastroenterology, vol. 106, no. 5, pp. 867-874, 2011.

[23] E. P. Saraga, D. Gardiol, and J. Costa, "Gastric dysplasia. A histological follow-up study," American Journal of Surgical Pathology, vol. 11, no. 10, pp. 788-796, 1987.

[24] M. J. Coma del Corral, F. J. Pardo-Mindan, S. Razquin, and C. Ojeda, "Risk of cancer in patients with gastric dysplasia: followup study of 67 patients," Cancer, vol. 65, no. 9, pp. 2078-2085, 1990.

[25] H. K. Koch, M. Oehlert, and W. Oehlert, "An evaluation of gastric dysplasia in the years 1986 and 1987," Pathology Research and Practice, vol. 186, no. 1, pp. 80-84, 1990.

[26] J. L. Whiting, A. Sigurdsson, D. C. Rowlands, M. T. Hallissey, and J. W. L. Fielding, "The long term results of endoscopic surveillance of premalignant gastric lesions," Gut, vol. 50, no. 3, pp. 378-381, 2002.

[27] M. Rugge, M. Cassaro, F. Di Mario et al., "The long term outcome of gastric non-invasive neoplasia," Gut, vol. 52, no. 8, pp. 1111-1116, 2003.

[28] K. C. R. B. Ramesar, D. S. A. Sanders, and D. Hopwood, "Limited value of type III intestinal metaplasia in predicting risk of gastric carcinoma," Journal of Clinical Pathology, vol. 40, no. 11, pp. 1287-1290, 1987. 
[29] S. Silva, M. I. Filipe, and A. Pinho, "Variants of intestinal metaplasia in the evolution of chronic atrophic gastritis and gastric ulcer. A follow up study," Gut, vol. 31, no. 10, pp. 10971104, 1990.

[30] T. Rokkas, M. I. Filipe, and G. E. Sladen, "Detection of an increased incidence of early gastric cancer in patients with intestinal metaplasia type III who are closely followed up," Gut, vol. 32, no. 10, pp. 1110-1113, 1991.

[31] J. M. Conchillo, G. Houben, A. de Brune, and R. Stockbrügger, "Is type III intestinal metaplasia an obligatory precancerous lesion in intestinal-type gastric carcinoma?" European Journal of Cancer Prevention, vol. 10, no. 4, pp. 307-312, 2001.

[32] L. Vannella, E. Lahner, and B. Annibale, "Risk for gastric neoplasias in patients with chronic atrophic gastritis: a critical reappraisal," World Journal of Gastroenterology, vol. 18, no. 12, pp. 1279-1285, 2012.

[33] M. Rugge, A. Meggio, G. Pennelli et al., "Gastritis staging in clinical practice: the OLGA staging system," Gut, vol. 56, no. 5, pp. 631-636, 2007.

[34] L. G. Capelle, A. C. de Vries, J. Haringsma et al., “The staging of gastritis with the OLGA system by using intestinal metaplasia as an accurate alternative for atrophic gastritis," Gastrointestinal Endoscopy, vol. 71, no. 7, pp. 1150-1158, 2010.

[35] M. Rugge, M. de Boni, G. Pennelli et al., "Gastritis OLGAstaging and gastric cancer risk: a twelve-year clinico-pathological follow-up study," Alimentary Pharmacology and Therapeutics, vol. 31, no. 10, pp. 1104-1111, 2010.

[36] M. Rugge, J. G. Kim, V. Mahachai et al., "OLGA gastritis staging in young adults and country-specific gastric cancer risk," International Journal of Surgical Pathology, vol. 16, no. 2, pp. 150-154, 2008.

[37] G. N. Stemmermann, "Intestinal metaplasia of the stomach. A status report," Cancer, vol. 74, no. 2, pp. 556-564, 1994.

[38] M. Rugge, M. Fassan, M. Pizzi, G. Pennelli, D. Nitti, and F. Farinati, "Operative Link for Gastritis Assessment gastritis staging incorporates intestinal metaplasia subtyping," Human Pathology, vol. 42, no. 10, pp. 1539-1544, 2011.

[39] M. Rugge, P. Correa, M. F. Dixon et al., "Gastric mucosal atrophy: interobserver consistency using new criteria for classification and grading," Alimentary Pharmacology and Therapeutics, vol. 16, no. 7, pp. 1249-1259, 2002.

[40] W. Yasui, K. Sentani, N. Sakamoto, K. Anami, Y. Naito, and N. Oue, "Molecular pathology of gastric cancer: research and practice," Pathology, Research and Practice, vol. 207, no. 10, pp. 608-612, 2011.

[41] C. O. Gigek, E. S. Chen, D. Q. Calcagno, F. Wisnieski, R. R. Burbano, and M. A. Smith, "Epigenetic mechanisms in gastric cancer," Epigenomics, vol. 4, no. 3, pp. 279-294, 2012.

[42] A. H. Corvalan and M. J. Maturana, "Recent patents of DNA methylation biomarkers in gastrointestinal oncology," Recent Patents on DNA and Gene Sequences, vol. 4, no. 3, pp. 202-209, 2010.

[43] C. Zhao and X. Bu, "Promoter methylation of tumor-related genes in gastric carcinogenesis," Histology and Histopathology, vol. 27, no. 10, pp. 1271-1282, 2012.

[44] A. O. O. Chan, S. K. Lam, B. C. Y. Wong et al., "Promoter methylation of E-cadherin gene in gastric mucosa associated with Helicobacter pylori infection and in gastric cancer," Gut, vol. 52, no. 4, pp. 502-506, 2003.

[45] A. O. O. Chan and A. Rashid, "CpG island methylation in precursors of gastrointestinal malignancies," Current Molecular Medicine, vol. 6, no. 4, pp. 401-408, 2006.
[46] T. Maekita, K. Nakazawa, M. Mihara et al., "High levels of aberrant DNA methylation in Helicobacter pylori-infected gastric mucosae and its possible association with gastric cancer risk," Clinical Cancer Research, vol. 12, no. 3, part 1, pp. 989-995, 2006.

[47] R. Ohki, J. Nemoto, H. Murasawa et al., "Reprimo, a new candidate mediator of the p53-mediated cell cycle arrest at the G2 phase," The Journal of Biological Chemistry, vol. 275, no. 30, pp. 22627-22630, 2000.

[48] B. G. Schneider, D. F. Peng, M. C. Camargo et al., "Promoter DNA hypermethylation in gastric biopsies from subjects at high and low risk for gastric cancer," International Journal of Cancer, vol. 127, no. 11, pp. 2588-2597, 2010.

[49] C. Bernal, F. Aguayo, C. Villarroel et al., "Reprimo as a potential biomarker for early detection in gastric cancer," Clinical Cancer Research, vol. 14, no. 19, pp. 6264-6269, 2008.

[50] N. S. Sapari, M. Loh, A. Vaithilingam, and R. Soong, "Clinical potential of DNA methylation in gastric cancer: a metaanalysis," PLoS One, vol. 7, no. 4, Article ID e36275, 2012.

[51] G. Carrasco, J. Diaz, J. R. Valbuena et al., "Overexpression of p73 as a tissue marker for high-risk gastritis," Clinical Cancer Research, vol. 16, no. 12, pp. 3253-3259, 2010.

[52] D. Faratian, "Systems pathology," Breast Cancer Research, vol. 12, supplement 4, article S4, 2010.

[53] A. I. Saeed, N. K. Bhagabati, J. C. Braisted et al., “TM4 microarray software suite," Methods in Enzymology, vol. 411, pp. 134-193, 2006. 


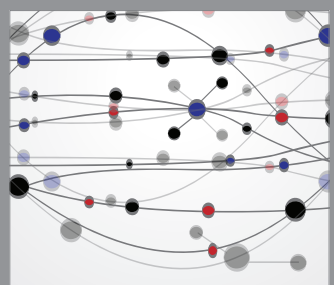

The Scientific World Journal
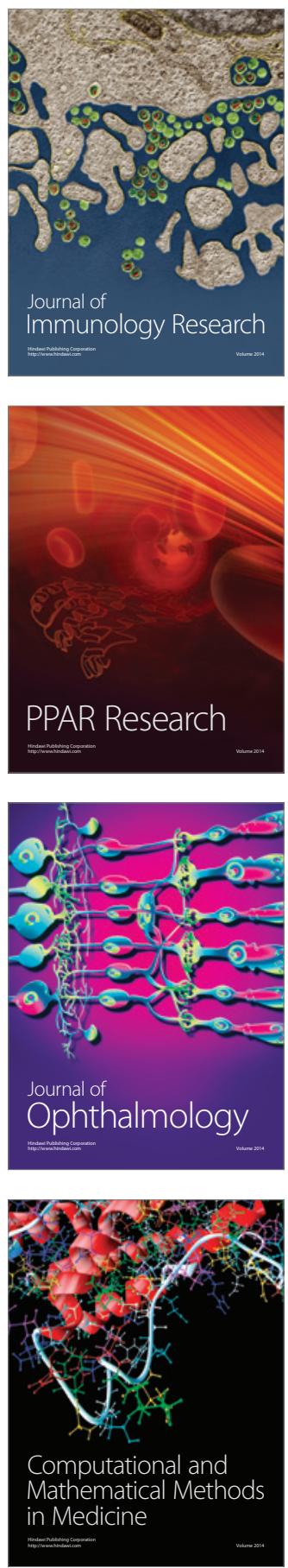

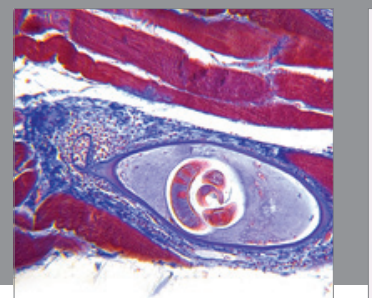

Gastroenterology

Research and Practice
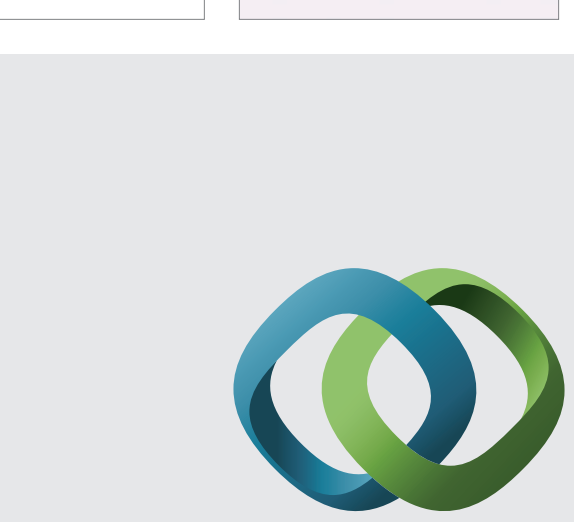

\section{Hindawi}

Submit your manuscripts at

http://www.hindawi.com
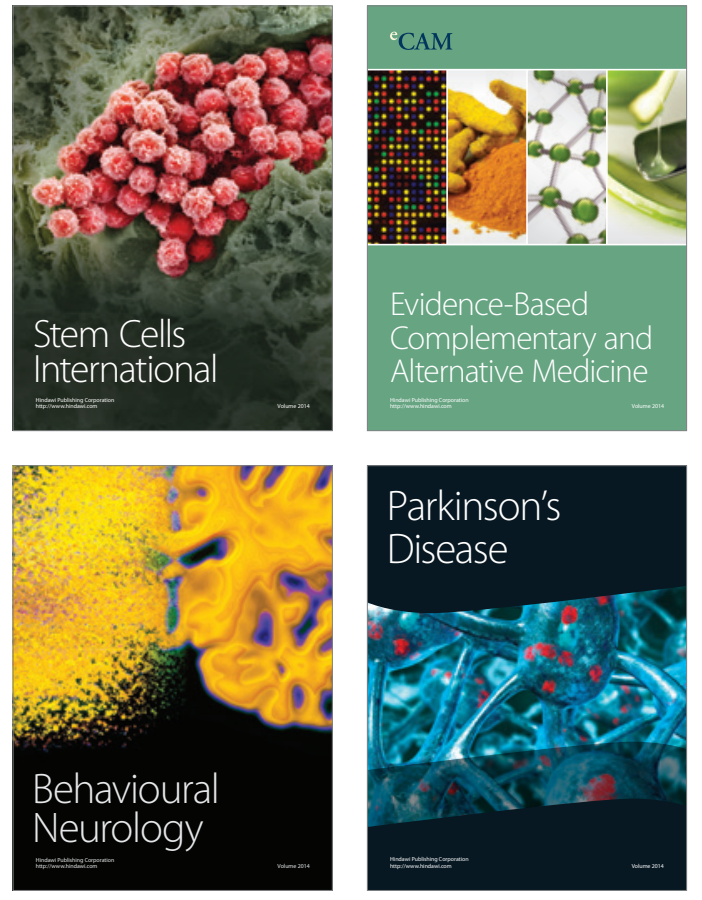
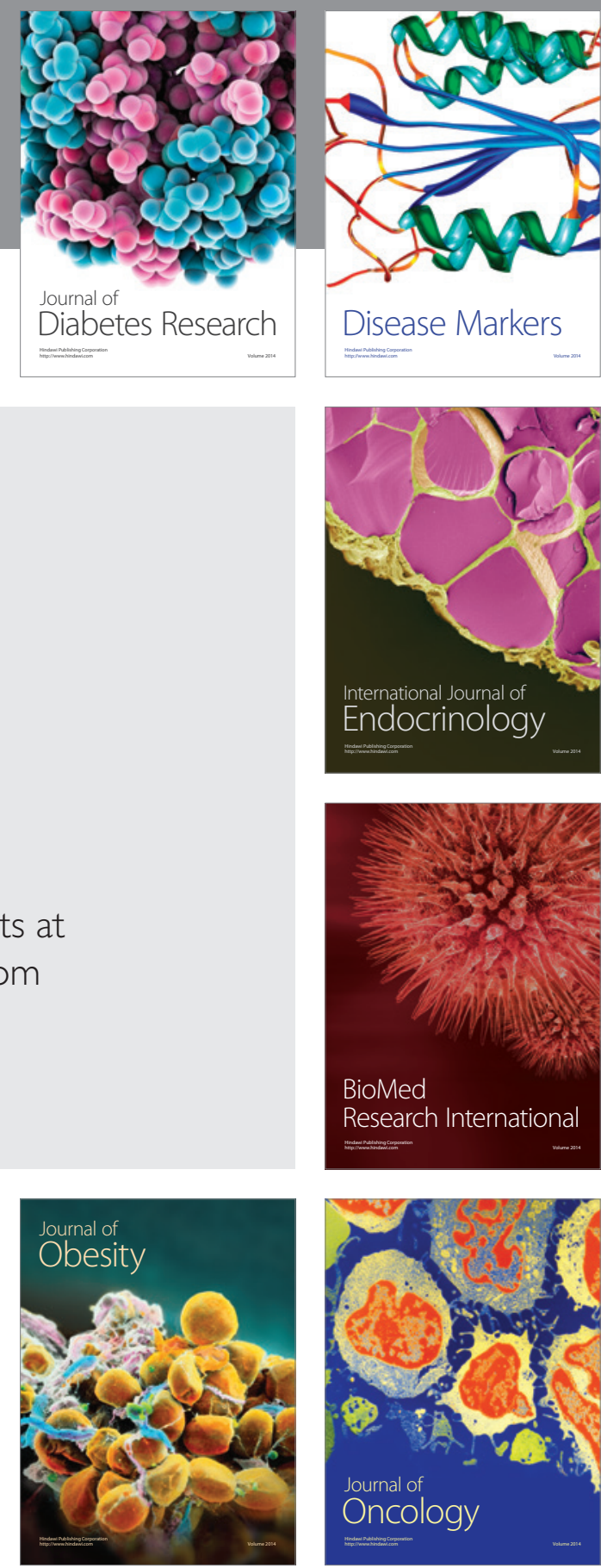

Disease Markers
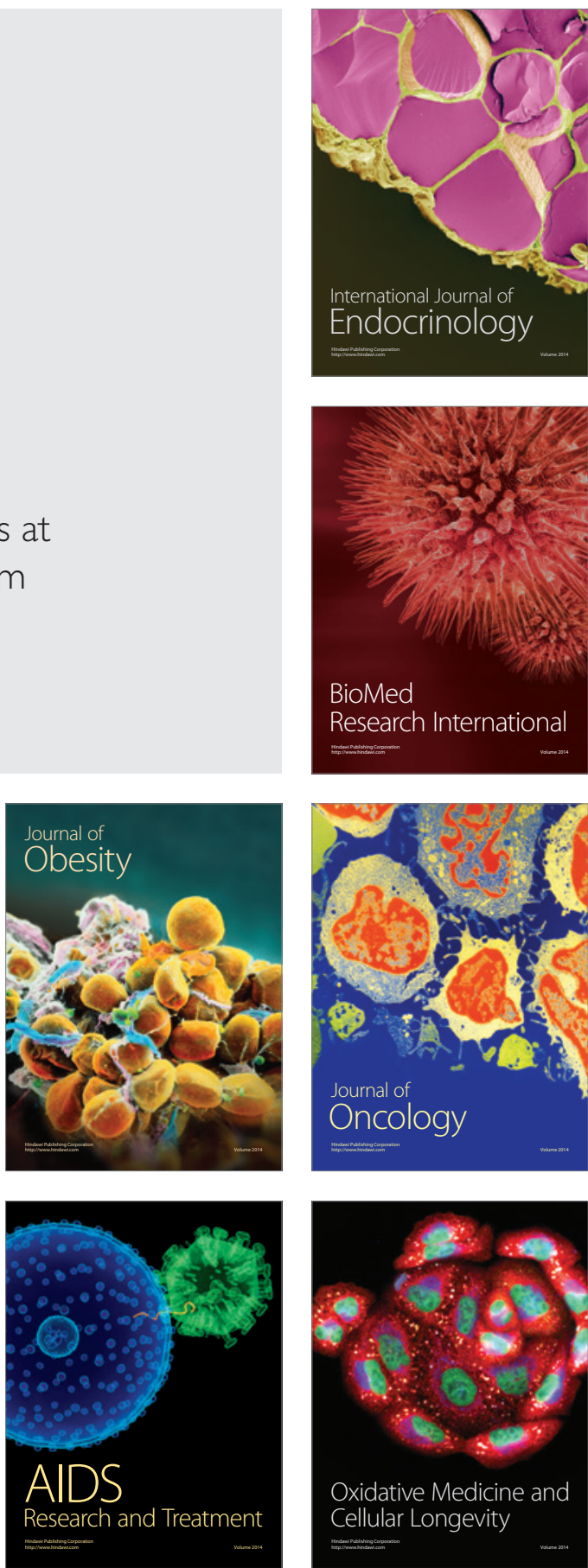